\title{
PERSPECTIVAS TEÓRICAS DE LA ADMINISTRACIÓN PÚBLICA
}

\section{THEORETICAL PERSPECTIVES OF THE PUBLIC ADMINISTRATION}

\author{
Lenín Javier Tobar \\ Universidad Central del Ecuador \\ ingltobar@yahoo.es \\ Luis Dávila Toro \\ Universidad Tecnológica Equinoccial \\ luisa.davila@ute.edu.ec
}

\begin{abstract}
Resumen
Este artículo tiene por objetivo seleccionar dos perspectivas teóricas de la Administración Pública para su análisis y definición de semejanzas y diferencias respecto de: Finalidades de la Administración Pública; Principales productos y sus características; Actores Relevantes en las políticas públicas; Resultados a los que se orienta, y Casos que puedan representarlos. Se escogió como perspectivas a la Nueva Gestión Pública vs Gobierno Abierto. Para la realización de esta tarea se utilizó el método bibliográfico, analítico y sintético, fijando como metodología la lectura de los diferentes postulados de cada perspectiva, tratando cada caso por separado en su análisis y síntesis. El trabajo permitió desprender que tanto en el enfoque de la Nueva Gestión Pública, como en el del Gobierno abierto se definen elementos en común desde el punto de vista de la organización del estado. Es interesante distinguir como es que en los dos casos centran sus esfuerzos en conseguir resultados en favor de la sociedad civil, las iniciativas privadas y todo el colectivo socio organizacional. Las diferencias son marcadas en cuanto a resultados, pues la esencia de la Nueva Gestión Pública concentra su atención como actor relevante al gobierno, siendo este, una organización que persigue un solo fin: una estructura técnica eficiente para proveer servicios. Por lo tanto produce un ethos precario de justicia: la justicia proviene de una organización técnica, no mediante organizaciones ni diálogo político. En cambio que para Gobierno Abierto sus esfuerzos se orientan a propiciar compromisos concretos de sus actores relevantes que provienen desde los gobiernos para promover la transparencia, aumentar la participación ciudadana en el debate y en la decisión de los asuntos públicos, combatir la corrupción y aprovechar las nuevas tecnologías para robustecer la gobernanza democrática y la calidad de los servicios públicos.
\end{abstract}

Palabras Clave: Administración Pública, Gobierno Abierto, Gestión Pública.

\begin{abstract}
This article aims to select two theoretical perspectives of the Public Administration for its analysis and definition of similarities and differences with respect to: Purposes of the Public Administration; Main products and their characteristics; Relevant actors in public policies; Results to which it is oriented, and Cases that can represent them. The New Public Management vs Open Government was chosen as perspectives. To carry out this task, the bibliographic, analytical and synthetic method was used, setting as a methodology the reading of the different postulates of each perspective, treating each case separately in its analysis and synthesis. The work made it possible to infer that both the New Public Management approach and the Open Government approach define common elements from the point of view of the state organization. It is interesting to distinguish how in both cases they focus their efforts on achieving results in favor of civil society, private initiatives and the entire socio-organizational collective. The differences are marked in terms of results, because the essence of the New Public Management focuses its attention as a relevant actor to the government, being this an organization that pursues a single purpose: an efficient technical structure to provide services. Therefore, it produces a
\end{abstract}


precarious ethos of justice: justice comes from a technical organization, not through organizations or political dialogue. On the other hand, for the Open Government, its efforts are aimed at propitiating concrete commitments of its relevant actors that come from the governments to promote transparency, increase citizen participation in the debate and in the decision of public affairs, fight corruption and take advantage of the new technologies to strengthen democratic governance and the quality of public services

Keywords: Public Administration, Open Government, Public Management

\section{Introducción}

En la práctica de la economía se manifiesta un dilema con relación a la capacidad óptima del sector público y a la eficiencia expuesta en el ejercicio de sus funciones. Centrándose en un primer momento la discusión en la selección al gestor adecuado capaz de gestionar los intereses de la sociedad: la función pública o la iniciativa privada. Las discrepancias de la utilidad en la sociedad del siglo XIX, y el no cumplimiento de la eficiencia de los mercados ayudaron a que el estado asumiese un papel más activo, intentado con su interposición rectificar las fallas del mercado (Ardila, 2011). Posteriormente, en la década de los 70 del siglo XX, inicia la preocupación por las ineficacias manifestadas en la realización de los programas públicos, surgiendo el planteamiento de los fallos de la intervención del estado, en la economía, atribuidos a la burocracia, la competencia política. La pérdida de confianza del sector público como principal gestor del bienestar de la ciudadanía viene acompañado de expresiones que reclaman y fuerzan a que los organismos públicos actúen aplicando principios de economía, eficiencia y eficacia. Es por ello que la Administraciones Públicas tanto locales como nacionales se han visto envueltas en procesos de generación, de cambio, a través de la adopción de nuevos modelos de acción y conducta, que permitan diseñar un nuevo método de operatividad pública o una nueva filosofía de actuación: la Nueva Gestión Pública (Villanueva, 2006).

No existe duda alguna, que internet ha revolucionado la cultura y, por lo tanto, la forma como opera la sociedad del momento (Dutton, 2011). Permitiendo que la concepción del Gobierno Abierto deje de ser una fantasía. Tratándose en realidad de un nuevo desarrollo tecnológico o de una novedosa manera de hacer lo que antes se hacía por otros medios. Es por esto que resulta un nuevo instrumento o el soporte de una filosofía distinta acerca de cómo gobernar y de cuál es el rol que desempeña el gobierno y los ciudadanos en la gestión pública y en sus resultados.

Esta nueva filosofía ha recibido el impulso concluyente generado por la disponibilidad de herramientas, que hacen posible el establecimiento de una comunicación de bidireccional entre gobierno y ciudadanía. No significando esto a que las TICs resulten una condición necesaria para imponer esta manera de hacer gestión. Concibiéndose más bien a un gobierno dispuesto a abrirse a la participación e involucramiento de los ciudadanos sin la mediación de herramientas informáticas (Hood C. , 1995). Permitiendo que el gobierno pueda ser considerado abierto por su sentidos de promoción e involucramiento en la gestión de organizaciones sociales y ciudadanos, sin que ello deba darse, necesariamente, a través de interacciones virtuales a través de las tecnologías actuales disponibles.

Es por lo anteriormente expuesto que tanto la revisión como el análisis de las principales teorías, visión y orientaciones que sustentan a los modelos teóricos de Nuevas Gestión Pública y Gobierno Abierto y el establecimiento de una comparación entre ambas visiones permitirá entender los motivos por las que ambas perspectivas teóricas , puedan ser consideradas en el contexto de la Administración Pública. 


\section{Metodología}

El método de investigación empleados está fundamentado en el enfoque explicativo, por medio de una revisión documental tanto de fuentes primarias como secundarias como parte esencial de un desarrollo investigativo científico, que permite observar y reflexionar sobre las diversas realidades, relacionadas con la Nuevas Gestión Pública y el Gobierno Abierto.

\section{Concepto Nueva Gestión Pública y Gobierno Abierto}

Primeramente se es importante considerar las definiciones correspondientes a Nueva Gestión Pública y Gobierno Abierto de modo de poder ir teniendo conocimiento acerca de lo que se aborda en cada concepto.

En el caso de la Nueva Gestión Pública se puede decir, que para muchos entendidos o expertos en la materia es un sistema, de conducción totalmente descentralizado, en el que se aplican instrumentos innovadores de gestión tales como: el controlling, el bechmarking y la gestión esbelta (lean managment), sirviendo también de instrumento de gestión de gobierno.

Siendo el Gobierno Abierto una orientación que permite de manera particular entender los procesos propios de gobierno, partiendo de las definiciones y principios de la transparencia y participación ciudadana. Esta perspectiva se ha venido conformando en recientes años producto de : a) la globalización ,específicamente con respecto a algunas dinámicas políticas nacionales y locales, de amplia trascendencia; b) el reconocimiento cada vez más creciente de que el gobierno no es la única esfera legítima capaz de atender y dar respuesta a los requerimientos de complejidad de las comunidades. c) la búsqueda de mecanismos político- administrativos alternativos a los tradicionales que permitan robustecer la legitimidad de los Estados.

Estos tres aspectos han influido el modo de cómo se deberían conformar las labores públicas y gubernamentales, representando un nuevo modelo de gobernanza. Lográndose a través del Gobierno Abierto la incorporación de la ciudadanía y las organizaciones de la sociedad civil en aquellos procesos de: planeación, ejecución y evaluación de las actividades gubernamentales, con el propósito de dar respuesta a muchas de las complejas demandas sociales de los países.

1. Caracterización de la Nueva Gestión Pública y el Gobierno Abierto

Es importante destacar y hacer mención de los principales elementos que caracterizan tanto a Modelo de Nueva Gestión Pública como el de Gobierno Abierto de modo que permita sentar parte de las bases para establecer una comparación entre ambas perspectivas actualizadas de cómo se conciben las practica publica hoy en día.

Dentro de las características que distinguen a la Nueva Gestión Pública se destaca:

- La Dirección está orientada al trabajo por competencias separadas entre los financiadores y los agentes prestadores de servicio.

- Los procesos de planeación, ejecución y evaluación de las acciones a seguir para la atención de las necesidades sociales se llevan a cabo y están centradas en el trabajo efectivo, eficiente y con calidad.

- Existencia de un una separación de la dirección, considerada estratégica, respecto a la dirección operativa.

- Atención de carácter igualitario de los prestadores de servicios independientes y de naturaleza pública, enmarcados por supuesto en la prestación de servicios y presupuestos de tipo global.

- La Nueva Gestión Pública está enfocada en procesos de innovación en la prestación de servicios, el cual permite optimizar las operaciones delegadas. 
En el caso del Gobierno Abierto a través de él se puede informar tanto las decisiones como acciones gubernamentales de forma inclusiva y transparente, innovadora manera de interrelación entre el gobierno y la ciudadanía. A través de los siguientes aspectos que lo caracterizan:

1. Establecimiento de canales de comunicación y contactación directa con la ciudadanía.

2. Ofrecimiento con altos niveles de transparencia y acceso a la información por medio de data abierta, reutilización de información estratégica del sector público y participación ciudadana en la construcción y ejecución de políticas de carácter público.

Generación de estrategias que permitan registrar la actividad de los gobiernos mediante procesos de documentación empleando las nuevas tecnologías.

3. Facilitación y acceso a la información e innovación de contenidos que puedan ser empleados por los usuarios.

4. Impacto en la calidad del rol del funcionario público.

5. Ejercicio de acciones y programas empleando criterio inclusivo de la ciudadanía en el diseño, ejecución y evaluación de las políticas públicas planteadas.

6. Fomento de acciones que permita llevar a cabo denuncias respectivas en cuanto a aquellas conductas carentes de ética por parte de los servidores públicas.

\section{Origen y Teorías de la Nueva Gestión Pública y el Gobierno Abierto}

En el caso de la Nueva Gestión Pública existen un conjunto de Teorías de las cuales se basa la Gestión Pública, las cuales se citan a continuación:

\subsection{La Teoría de la Elección Pública o «Public Choice»}

La teoría de la elección u opción pública, busca enlazar el contexto económico con la política a través del Estado, producto de la suma de voluntades de manera individual, que permitan saber cuáles son los factores que determinan las políticas de Estado de una diversidad de opciones. Esta teoría abarca dos áreas: a ) La Elección pública Positiva, que se encarga de estudiar las decisiones tanto públicas como colectivas de los agentes políticos y b) La Economía Política Constitucional , cuyo propósito es desarrollar un marco constitucional que contribuya a la disminución del poder político respecto a la sociedad civil.

Esta teoría está basada en la necesidad en establecer relaciones contractuales con el exterior con el propósito de aumentar la eficiencia y evitar las ineficiencias de la Burocracia.

\subsection{Teoría de los Costes de Transacción}

La Teoría de los Costes de Transacción tiene como objetivo identificar las fuentes de los costos de transacción (características propias de una transacción) especificando el mecanismo de gobierno, que pueda regularizar con mayor eficiencia dicha transacción con el fin de lograr abaratar costos. Entendiéndose que la transacción como concepto, es el intercambio tanto de bienes como de servicios en una organización, intercambio de propiedad por bienes y servicios. Costos que pueden estar asociados a factores como: la búsqueda de personas o bienes, los derechos de transferencia, la negociación de términos de acuerdos, entre otros.

Willamson (Barzelay \& Michael, 2003) identifica los costes de transacción asociado a la firma de los contratos, el control de resultados y comportamiento de las partes contractuales. Costes que varían, de acuerdo a las características de las decisiones de los gestores que participan en la transacción y con los objetivos del mercado. Así la estructura organizacional optima, lleva a cabo dicho proceso en 
función de los costos asociados a ella, lo que conduce a la Administración Pública a incorporar tanto condiciones análogas a las del mercado como elementos de control .

\subsection{La Teoría de la Agencia}

La Teoría de la Agencia, representa un método de perfeccionamiento a nivel empresarial, a través de un sistema de subcontratación, de diferentes tareas por parte de una organización, con el propósito de hallar una adecuación correcta de dicho trabajo. La Teoría de la Agencia es un pensamiento empresarial que surge en el año 1973 de la mano de Ross (Barzelay \& Michael, 2003):

"Cada uno de los sujetos de una relación de agencia tiene sus propios intereses y ambos intentan maximizar la utilidad."

La Teoría de la Agencia puede ser implantada a varios puntos de la actividad empresarial de forma múltiple. Siendo un modelo en el que se orientan algunos acuerdos empresariales o laborales para lograr un propósito mutuo que aporte beneficios a las partes involucradas.

En el ámbito público la definición de principal y agente es compleja al encontrarnos en presencia de múltiples figuras. Mayston se cuestiona quien es el principal

en dicha relación, descubriendo tres posibles respuestas a dicha pregunta: el electorado - a

través de los procesos democráticos de elecciones y los controles parlamentarios-; los consumidores de los servicios públicos; y los políticos del gobierno central en el poder. Albi et al. (1997, p. 90) establecen relaciones de agencia superpuestas entre distintos niveles de la gestión pública, tales como ciudadanos, parlamentarios, gobierno, agencias y empleados públicos; ampliadas por Pina Martínez y Torres Prada bajo la teoría de la red contractual a prestamistas, sindicatos y otros agentes legítimamente interesados (Barzelay \& Michael, 2003).

Es por ello que se entiende que en la Administración Pública existen dos niveles básicos de relación de agencia:

a) La generada por la delegación de responsabilidad representada por la cadena : Ciudadano Políticos Gestores, Delegación de responsabilidad que se produce en todos los órganos de la Administración Pública.

b) La relación Inter-administraciones: son las relaciones denominadas luchas de poder entre las diferentes administraciones territoriales con el propósito de obtener mayor supremacía a través de la sustracción de competencias estatales y por ende la desviación de recursos financiero, sin pensar en ocasiones en el bienestar social, sino más bien en los intereses de aumentar el poder político.

\subsection{Teoría de la Nueva Gestión Pública}

La Teoría de la Nueva Gestión Pública representa una corriente teórica que surgió para dar respuesta y enfrentar la problemática asociada al modelo burocrático. También llamado paradigma postburocrático o Administración Pública Gerencial, propone la introducción en el sector público, de componentes propios de la empresa privada, mediante el un estado orientado al trabajo efectivo de sus funciones y prestación de servicios, empleando para ello conceptos del liderazgo corporativo inspirados en los postulados neoliberales a nivel económico, cuyo enfoque principal es el aumento de la eficiencia. 
Según la mayoría de los autores estudiados (Arellano \& Cabrero, 2005) (Barzelay \& Michael, 2003) (CLAD, 1998) (Boston \& Al, 1996), las principales características de la teoría de la NGP son: la descentralización, el gerencialismo, la costo-eficiencia, los incentivos a la competitividad, la desregulación, la reducción del tamaño del gobierno mediante cierres o privatizaciones (tercerización) y de la intervención de este en la economía.

La Nueva Gestión Pública no va a descubrir al mercado como recurso para el sector público, sino que enfatiza sus valores y produce un nuevo tipo de relación entre ambos. En este modelo, el ciudadano solamente puede votar a sus representantes para que gobiernen, que se ocuparán a través de terceros de gestionar los servicios públicos, por lo que el ciudadano se verá aislado de los espacios de consulta, decisión y control.

\section{Gobierno Abierto}

El Gobierno Abierto existe desde el siglo XVI cuando fue establecido en Suecia y los datos gubernamentales fueron hechos públicos como un derecho constitucional; así, la gente pudo tener acceso a esta información (Calderón \& Lorenzo, 2010).

Aproximadamente en el año 1989, luego de la Caída del Muro de Berlín. En ésta época histórica se comienza a considerar la necesidad de emplear las tecnologías de información y comunicación con el objetivo de impulsar la idea de gobierno transparente como principal promotor de la redición de cuentas y por ende la apertura de los datos (Chapman \& Hunt, 1987) (Dassen \& Cruz Vieyra, 2012).

Cabe destacar que los aspectos teóricos -conceptualización, modelos teóricos, construcción de teoríadel gobierno abierto, se expondrán siguiendo tres grandes direcciones teóricas: 1) libertad de información, 2) información gubernamental como herramienta, y 3) datos abiertos

\section{1era Orientación: La Libertad de Información}

El Gobierno Abierto es fruto de discusiones teóricas, por el derecho que tiene todo ciudadano a tener acceso a la información de los entes de gobierno. Siendo el punto central de ello, la democracia como sistema de gobierno, ya que ésta libertad de información es parte de las libertades otorgadas por la misma. En este sentido, Richardson (1973) afirma que la libertad de información es un componente básico del proceso democrático; de acuerdo con esta idea llamada "derecho de saber" (right to know), se convierte en lo opuesto al secreto gubernamental (Boston \& Al, 1996).

Bennett (1985), propone una ley de libertad de información que ayude a la apertura de registros gubernamentales y facilite su acceso por parte de los ciudadanos (Calderón \& Lorenzo, 2010).

Morris et al. (1981) inicia el estudio metódico con relación a la libertad de información y el debate entre el secreto gubernamental y el acceso a la información. Otros aportes con relación a dicha libertad fueron llevados a cabo por Perritt (1996), quien lleva a cabo una revisión de las políticas públicas con el objetivo de abrir la información del gobierno.

Otros académicos llevaron a cabo investigaciones similares, considerando casos tanto de gobiernos locales como federales. Si embargo no es hasta el año 2000 cuando el auge de la computación y el uso de las TIC, dan inicio a una nueva dinámica a la llamada libertad informativa. Existen muchos otros estudios que se desarrollaron en muchas otras naciones con respecto a éste tema y con relación a éste grupo de ideas relacionadas con la libertad de información se puede decir que ésta tendencia teórica se ha convertido en un antecedente real en lo que hoy se denomina gobierno abierto y que está relaciona además con la transparencia. Siendo la libertad de información un factor de importancia y de impacto que permite la idea de un gobierno abierto y transparente respecto a la relación con la ciudadanía, 
traduciéndose todo ello en libertad democrática, lo cual permite establecer el concepto de gobierno abierto.

\section{2da Orientación: La Información Gubernamental como Herramienta}

La segunda visión teórica considera la información del gobierno como un instrumento que facilita la toma de decisiones. Parte de la idea de la libertad de datos. La demanda de información desde los ciudadanos, la relación de confianza gobierno-ciudadanos y la relación entre gobierno-apertura de información son actividades que permiten explicar la implantación de la transparencia producto de los datos gubernamentales, así como la forma en que dicha información se vincula entre si y es organizada (GOBERNA, Escuela de Política y Alto Gobierno, 2013)

Curtin y Meijer (2006) proponen lo siguiente para hacer posible esta idea de información abierta: 1) La transparencia aumenta la legitimidad, 2) la transparencia produce legitimidad, y 3) genera una legitimidad social.

Otro elemento de esta corriente donde el gobierno abierto puede ser entendido como una herramienta de información es considerarlo como una fuente que genera valor público (Harrison et al., 2011).

Un último componente de dicha corriente es el que se refiere a tratar de modelar el impacto del gobierno abierto y el contexto social. Un esfuerzo en esta dirección es el realizado por Scholl y LunaReyes (2011), quienes a partir del uso de la dinámica de sistemas lograron proponer un modelo que combinara el uso de la información gubernamental, la participación y la colaboración de los ciudadanos.

Finalmente se entiende que el gobierno abierto es una herramienta que impulsa la cooperación, el debate, la construcción conjunta de ideas, la participación ciudadana y la colaboración entre el ciudadano y el gobernante en turno (GOBERNA, Escuela de Política y Alto Gobierno, 2013) Alentando los procesos democráticos y de rendición de cuentas, (GOBERNA, Escuela de Política y Alto Gobierno, 2013) menciona que es "el instrumento más prometedor para repensar y acentuar el concepto de ciudadanía en una sociedad democrática".

\section{3ra Orientación: Los Datos Abiertos}

Esta tercera visión teórica que se ha venido desarrollando con rapidez en los últimos tiempos, y cuya agrupación ha impactado al gobierno abierto, en lo referente a la apertura de los datos como un complemento necesario para alcanzar la apertura por parte del gobierno. Siendo los datos una expresión muy elemental de apertura de información- Además de que los datos pueden ser editados, sistematizados y analizados por la ciudadanía detalladamente, tener una visión más extensa sobre las cuestiones públicas.

Incluso estos datos abiertos permiten impulsar la coproducción, colaboración y participación de los ciudadanos en los procesos públicos al proveer a los funcionarios públicos de nuevos recursos información, que muestren una orientación o visión distinta, alternativas o propuestas diferentes s a la visión gubernamental.

En cuanto a ésta dirección resulta que, es la forma como los ciudadanos usan los datos abiertos para resolver sus propias necesidades de información. En este sentido, las investigaciones se vinculan directamente al uso de redes sociales y datos abiertos. Por ejemplo, el proyecto SOMUS propone diversas herramientas para abrir los datos gubernamentales y aprovecharlos (Calderón \& Lorenzo, 2010). O bien, el uso de la herramienta de crowdsourcing (multitudes inteligentes) para impulsar la participación de los ciudadanos.

\section{Fines de la Nueva Gestión Pública y el Gobierno Abierto}


Con relación a la Nueva Gestión Pública se busca satisfacer las necesidades de la ciudadanía, por medio de una gestión pública tanto eficaz como eficiente, es por ello que los servicios prestados por el Sistema de Administración Pública deben realizarse por medio de actividades con mayor calidad, en un marco de sistemas de control que promuevan la transparencia en los procesos de elección tanto de planes como de resultados, así como de la participación ciudadana como una manera de ejercer la administración pública. Siendo la NGP un modelo aplicado en el contexto del sistema privado pero que busca extrapolarse a la institucionalidad pública.

En síntesis la Nueva gestión Pública se fundamenta en:

1. La formulación estratégica de políticas de desarrollo y gestión.

2. La sucesiva eliminación del modelo burocrático, hacia otro en base a resultados alcanzados.

3. La creación de valor público,

4. El desarrollo institucional adecuado al Estado.

5. El mejoramiento de las conquistas macroeconómicas e igualdad social.

La OCDE (OECD, 2016) considera que esta nueva forma de gestión de la Administración Pública se caracteriza por las líneas maestras que se exponen a continuación:

- Desregulación.

- Énfasis en las responsabilidades de los gestores y motivación para la mejora.

- El reforzamiento de las capacidades estratégicas del centro, junto con la reorganización e implantación de la función pública en la gestión y las reformas.

- Gestión más orientada hacia el cliente.

- La introducción de la competencia y el mercado.

- Utilización de métodos de evaluación y técnicas de gestión aplicadas en el ámbito empresarial.

Con respecto al Gobierno Abierto, representa un fenómeno de reciente aplicación, que ya es conocido a lo largo de éste artículo, se basa en las de las tecnologías de la información y de la comunicación (TIC) en la gobernanza pública, con una enorme trascendencia en pro de mejorar y potencializar la participación ciudadana en las cuestiones públicas y su implicación en la sociedad, en los procesos de toma de decisiones políticas.

Dutton (Dutton, 2011) plantea que, en el siglo XVIII, la tecnología de aquel momento permitió que los medios de comunicación fuesen reconocidos como el cuarto poder en las sociedades liberales democráticas y así permanecieron hasta la llegada de las nuevas tecnologías de la información y de la comunicación.

El creciente uso de internet permite a las personas conectadas en red acceder a fuentes alternativas de información y otros recursos, diferentes a las fuentes tradicionales de información (medios de comunicación de masas, bibliotecas, catálogos de información, etcétera). Este acceso a la información hace posible - dice Dutton- que las personas conectadas en red utilicen este espacio virtual para ir más allá de los límites de las instituciones, abriendo con ello nuevas formas de aumentar la rendición de cuentas de los políticos, de la prensa, de los expertos y otros focos de poder e influencia.

Por ello, este autor considera que las tecnologías digitales han generado la aparición de un nuevo escenario público por medio de una red en la que cientos de ciudadanos se comunican y posibilitan una nueva fuente de información para la fiscalización y la rendición de cuentas del gobierno.

La sucesión de diferentes herramientas tecnológicas aplicadas a la política ha dejado claro que el principal interés no está en las propias herramientas, sino en la información y en los flujos de 
comunicación que estas permiten, con miras a aumentar la transparencia informativa del gobierno, la rendición de cuentas y la participación ciudadana para recuperar la confianza de los ciudadanos.

\section{Conclusiones}

Tanto en el enfoque de la Nueva Gestión Pública, como en el del Gobierno abierto se definen elementos en común desde el punto de vista de la organización del estado. Es interesante distinguir como es que en los dos casos centran sus esfuerzos en conseguir resultados en favor de la sociedad civil, las iniciativas privadas y todo el colectivo socio organizacional. Las diferencias son marcadas en cuanto a resultados, pues para la Nueva gestión Pública es que el gobierno debe preocuparse no solo por gastar el dinero y llevar a cabo procedimientos, sino básicamente por ofrecer resultados hacia el área social, asegurando la universalidad de los servicios de salud y educación, y hacia la creación de instrumentos que ayuden a promover el desarrollo económico, garantizando las condiciones macroeconómicas favorables a la inversión privada y al aumento de la competitividad sistémica del país, mediante políticas sólidas en los sectores de ciencia y tecnología y del comercio exterior. (CLAD, 1998)

Así para el Gobierno abierto su prioridad como resultado es fortalecer la democracia al permitir el escrutinio público, proporcionando un baluarte contra la acumulación excesiva de riqueza o el poder en manos de unos pocos y por brindar mayores oportunidades para la participación de la ciudadanía. Muchas de las políticas impulsadas para una mayor apertura reconocen explícitamente la contribución que éstas medidas tienen en la mejora de la gobernanza democrática. (Concha \& Naser, 2012)

\section{Bibliografía}

Ardila, H. (2011). Clientelismo y tradición burocrática son amenazas para el buen gobierno. Portafolio, 30-32.

Arellano, D., \& Cabrero, E. (2005). La Nueva Gestión Pública y su teoría de la organización: ¿son argumentos antiliberales?Justicia y equidad en el debate organizacional público. Gestión y Política Pública, 599-618.

Barzelay, \& Michael. (2003). La nueva gerencia pública. Invitación al diálogo cosmopolita. Gestión y Política Pública, vol. XII, núm. 2, ii semestre, 2003, 241-251.

Boston, \& Al, e. (1996). Public Management: The New Zealand Mode. Oxford: Oxford University Press.

Calderón, C., \& Lorenzo, S. (2010). Open Government / Gobierno Abierto. Madrid: Algón Editores, España.

Chapman, R., \& Hunt, M. (1987). Open Government. A study of the prospects of open government within the limitations of the British political system. Routledge, London.: Croom Helm.

CLAD. (1998). Nueva Gestión Pública en América Latina. Reforma y Democracia N 39.

Concha, G., \& Naser, A. (2012). El desafío hacia el gobierno abierto en la hora de la igualdad. Santiago de Chile: CEPAL. 
Dassen, N., \& Cruz Vieyra, J. (2012). Gobierno abierto y transparencia focalizada : tendencias y desafíos para América Latina y el Caribe. Washington: BID.

Dutton. (2011). Una visión más amplia de Internet en el aprendizaje. En J. W. MORAVEC, Aprendizaje Invisible. Hacia una nueva ecología de la educación. COBO,.

GOBERNA, Escuela de Política y Alto Gobierno. (2013). EL GOBIERNO ABIERTO Y LOS DESAFÍOS. Madrid: Fundación José Ortega y Gasset-Gregorio Marañón.

GOBERNA, Escuela de Política y Alto Gobierno. (2013). Los desafios del Gobierno Abiero en América Latina. Madrid: Fundación José Ortega y Gasset-Gregorio Marañón.

Hood, C. (1991). A public Management for all seasons. Public Administration N 69, 50-62.

Hood, C. (1995). The "new public management" in the 1980s: Variations on a theme. Accountig,Organization and Society, 93-109.

Ingraham, P., \& Rosenblon, D. (1992). The Promise and paradox of civil service reform. Pittsburgh: University of Pittsburgh Press.

Manin, B. (1998). Los principios del gobierno representativo. Madrid: Alianza.

OECD. (2016). OECD Public Governance Reviews Open Government in Costa Rica. Paris: OEDC Publishing.

Villanueva, L. (2006). Gobernanza y gestión pública. México: Fondo de Cultura Económica. 